\title{
Web Applications Supporting the Development of Models of Chagas' Disease for Left Ventricular Myocytes of Adult Rats
}

\author{
Caroline Mendonça Costa, Ricardo Silva Campos, Fernando Otaviano Campos, \\ and Rodrigo Weber dos Santos \\ FISIOCOMP, the Laboratory of Computational Physiology, \\ Dept. of Computer Science and Master Program in Computational Modeling \\ Federal University of Juiz de Fora \\ Campus Martelos, \\ Juiz de Fora, MG 36036-330, Brazil \\ mendonca.carol@yahoo.com.br, ricardosilvacampos@terra.com.br, \\ fcampos1981@gmail.com, rodrigo.weber@ufjf.edu.br \\ http://www.fisiocomp.ufjf.br
}

\begin{abstract}
Chagas' Disease is an endemic infection in many areas of South and Central America that may cause a fatal type of myocarditis. In the acute phase of the disease, ventricular extrasystoles and tachycardia have been reported. Experiments with cardiac myocytes in the acute stage of Chagas' Disease have suggested a depression of $\mathrm{I}_{t o}$, the $\mathrm{Ca}^{2+}$-independent $\mathrm{K}^{+}$Transient Outward current. In this work we use computational models of left ventricular myocytes of adult rats to qualify the effects of Ito reduction that appear during the acute phase of Chagas' Disease. The simulations are carried out by Web applications based on the CellML language, a recently emerged international standard for the description of biological models. The computational framework supports the development of mathematical models, simulations and visualization of the results. Our preliminary simulation results suggest that the reduction of $\mathrm{I}_{t o}$ density elicits modifications of electrophysiological mechanisms that are strongly related to the phenomena of arrhythmia.
\end{abstract}

Keywords: Cardiac Modeling, Web-based Simulations, Problem Solving Environment, Myocarditis, Arrhythmia, Chagas' Disease.

\section{Introduction}

Chagas' Disease is an endemic infection in many areas of South and Central America causing a distinctive, often fatal myocarditis. Approximately 10 to 20 million individuals are infected, and 50,000 deaths annually are associated with this disease [1].

In the acute period ventricular extrasystoles and tachycardia have been reported in the cases of acute myocarditis 223. Experiments with cardiac myocytes in the acute stage of Chagas' Disease 4] have suggested a depression of $\mathrm{I}_{t o}$, 
the $\mathrm{Ca}^{2+}$-independent $\mathrm{K}^{+}$Transient Outward current in canine hearts. Animal models of myocarditis have also reported the depression of $\mathrm{I}_{t o}$ in rat heart. The availability of many different genetically altered mouse models of known defects in the human cardiovascular system, the recent completion of the rat genome [5] and improvements in methods for genetically modifying rat progeny [6] create a strong demand for a more quantitative understanding of murine cardiovascular physiology.

In this work we use computational models of the left ventricular myocytes of adult rats to qualify the effects of the $\mathrm{I}_{t o}$ reduction that appear during the acute phase of Chagas' Disease. Due to the multi-scale and multi-physics nature of these biological models, their development becomes particularly challenging, not only from a biological or biophysical viewpoint, but also from a mathematical and computational perspective. A Web-based computational framework that provides support for cardiac electrophysiology modeling is under development [7]8. Such framework integrates different tools and allows one to bypass many complex steps during the development and use of cardiac models that are based on systems of ordinary differential equations. The implementation of cardiac cell models is supported by a code generator named AGOS [7] that generates $\mathrm{C}++$ code by translating models described in the CellML language [9], a recently emerged international open standard for the description of biological models. The generated code allows one to manipulate and solve the mathematical models numerically. The set up and use of the computational models is supported by a user-friendly graphical interface that offers the tasks of simulation configuration, execution in a computer cluster, storage of results and visualization. All these tools are integrated in a Web portal [10. The Web portal allows one to develop and simulate cardiac models efficiently via this user-friendly integrated environment. As a result, the complex techniques behind cardiac modeling are taken care of by the Web distributed applications.

A series of in-silico experiments were performed in order to better understand how the depression of $\mathrm{I}_{t o}$ relates to the reported extrasystoles and tachycardia in the acute phase of the disease. The simulations focused on obtaining insights to better describe three important landmarks of arrhythmia: APD (Action Potential Duration) restitution curves; dispersion of repolarization; and the dynamics of intracellular $\mathrm{Ca}^{2+}$ concentration. The APD restitution curve seeks an indication of pro-arrhythmic behavior. During pacing and reentrant cardiac arrhythmias, APD restitution slope has been shown to be an important determinant of wave stability. In addition of promoting APD alternans, a steep (modulus greater than 1) APD restitution slope can promote breakup of electrical waves into a fibrillation-like state [1]. It is well known that amplification of dispersion of repolarization underlies the development of life-threatening ventricular arrhythmias [12. The dispersion of repolarization is influenced by the dispersion of APDs of the ventricular myocytes and the propagation velocity of the depolarization wave. In this work we have taken the difference between endocardial and epicardial APDs as an indication of transmural APD dispersion. Finally, alterations in intracellular calcium handling play a prominent role in the generation 
of arrhythmias in the failing heart. Abnormal intracellular calcium dynamics are usually correlated to the occurrence of Early (EAD) and Delayed (DAD) Afterdepolarization, i.e. the strong candidates for triggers of arrhythmia 13 .

In this work, we use a modified version of the Pandit et al [14 model to simulate action potentials (APs) in adult rat left ventricular myocytes. The mathematical description of the Pandit model was obtainned from the CellML repository 15. Our Web Portal supported the modification of the model, the simulation of many experiments and the visualization of results. Our simulation results suggest that the reduction of $\mathrm{I}_{t o}$ density observed during the acute phase of Chagas' Disease elicits the modification of many important electrophysiological mechanisms and features at the cellular level. The reduction of $\mathrm{I}_{t o}$ was followed by $\left[\mathrm{Ca}^{2+}\right] \mathrm{i}$ overload and promoted the occurrence of EADs, acting thus in a pro-arrhythmic fashion. However, reduction of $\mathrm{I}_{t o}$ also decreased the transmural dispersion of APDs, indicating an anti-arrhythmic response. Therefore, the observed modifications do not converge to the same direction in what concerns the prevention or induction of arrhythmia.

\section{Methods}

We have used a modified version of the Pandit et al. 14] model to simulate action potentials (APs) in adult rat left ventricular myocytes. The Pandit model was used to simulate both epicardial and endocardial myocytes as described in 14. The original model exhibits Early Afterdepolarizations (EAD) at pacing rates greater than $2 \mathrm{~Hz}$ due to insufficient inactivation and excessive reactivation of the L-type $\mathrm{Ca}^{2+}$ current $\mathrm{I}_{C a, L}$ [16. To solve these limitations the modifications proposed in [16] were implemented to avoid the occurrence of EAD at physiological rates: the time constant of fast inactivation related to the $\mathrm{Ca}^{2+}$-independent $\mathrm{K}^{+}$Transient Outward Current $\mathrm{I}_{t o}$ was reduced by $5 \%$; and the inactivation time constant $\mathrm{f} 11$ of $\mathrm{I}_{C a, L}$ was modified according to [16] in order to accelerate its kinetics.

These modifications were performed on the Web via a CellML editor which is one of the applications of a Web-based computational framework that provides support for cardiac electrophysiology modeling [78]. In addition, all simulations and the visualization of results were performed on the web with the support of our computational framework. After applying the above modifications to the original CellML model, the AGOS tool [7] translated the new description to an Application Program Interface (API) which is an object oriented $\mathrm{C}++$ code. Functions are created for initialization of parameters like the number of iterations, discretization interval and initial values of variables. There are functions for the numerical solution of the system of ordinary differential equations and for results storage. In addition, the API offers functions which allow the automatic creation of model-specific interfaces. This feature is used by another web application that enables one to set any model initial condition or parameter of the model, displaying their actual names, as documented in the CellML input file, as shown in Figure 1. The Web server is currently connected to a small cluster via the SGE engine [17] which enables the simulations to be executed. 


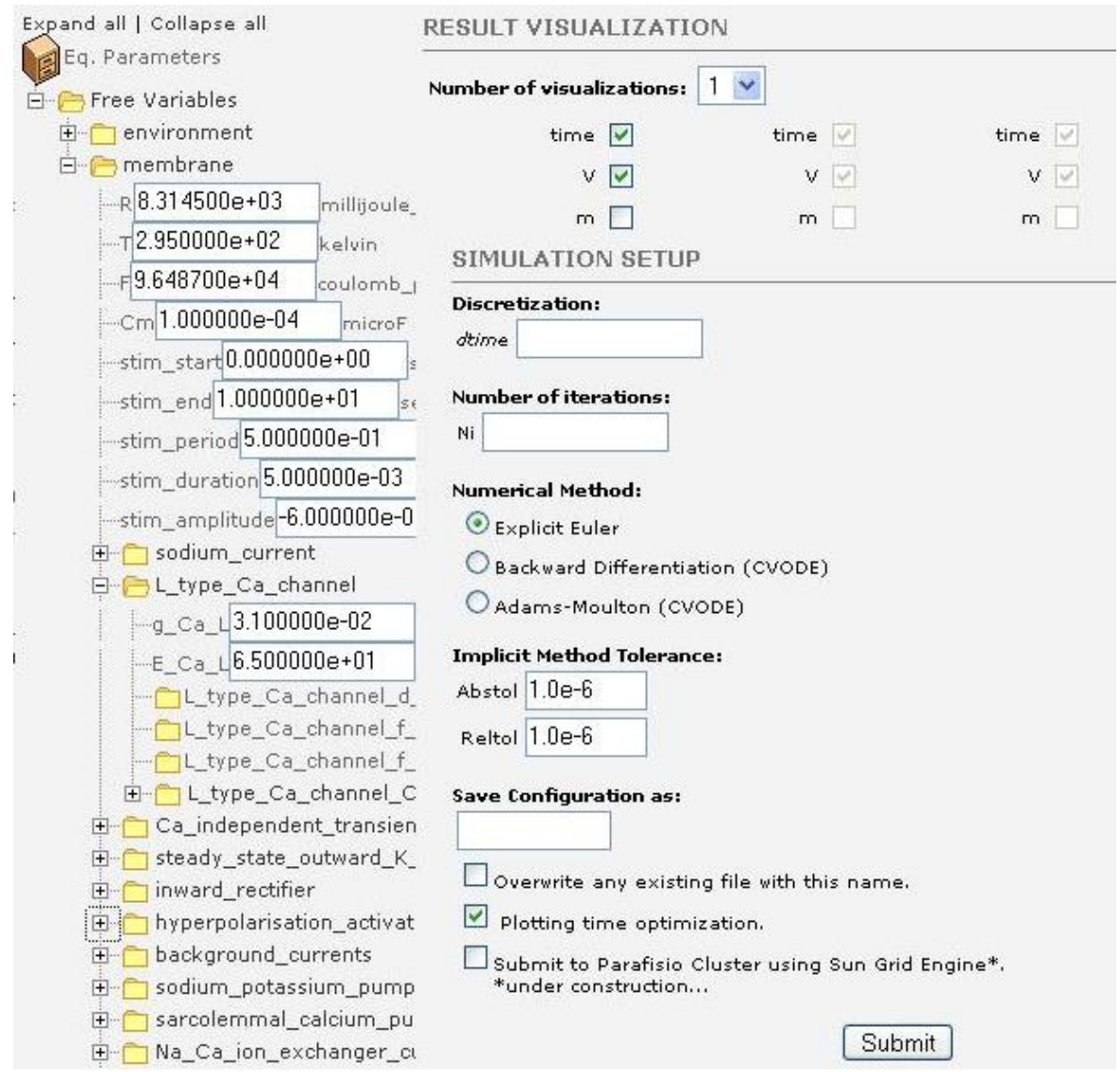

Fig. 1. Web form automatically generated by the AGOS tool for the Pandit model

The in-silico myocyte based on the Pandit model was stimulated by injecting a transmembrane current of $0.6 \mathrm{nA}$ during $5 \mathrm{~ms}$. A train of stimuli was simulated in order to reproduce the dynamic restitution protocol described in [18]. Cells were initially paced at a constant BCL (Basic Cycle Length) of $150 \mathrm{~ms}$. After 50 stimuli pacing was stopped and action potential duration (APD90) and the maximal upstroke velocity $(\max d v / d t)$ were measured. APD90, APD at $90 \%$ of repolarization, was calculated using the difference between the activation time and repolarization time. Activation times were obtained as the time of maximum rate of rise of the simulated transmembrane potentials. Repolarization times were calculated as the time of crossing a level corresponding to $90 \%$ of repolarization to the transmembrane resting potential. Pacing was then restarted at a longer BCL. At every 50 stimuli BCL was increased by $25 \mathrm{~ms}$ for the simulation with BCLs ranging from $150 \mathrm{~ms}$ to $300 \mathrm{~ms}$; by $50 \mathrm{~ms}$ for BCLs ranging from $300 \mathrm{~ms}$ to $500 \mathrm{~ms}$; by $250 \mathrm{~ms}$ from $500 \mathrm{~ms}$ to $1000 \mathrm{~ms}$; and by $500 \mathrm{~ms}$ from $1000 \mathrm{~ms}$ to $3000 \mathrm{~ms}$. 


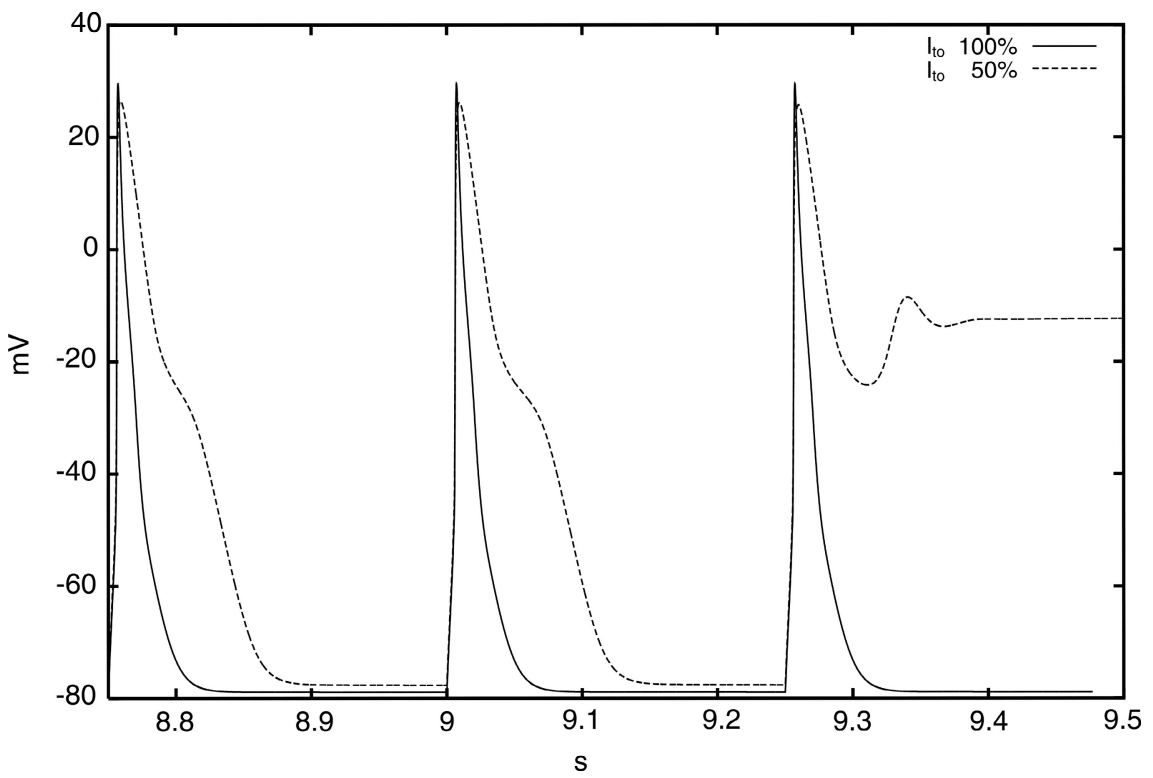

Fig. 2. Epicardial AP paced at a constant BCL of $250 \mathrm{~ms}$ for $50 \%$ reduction and control $\mathrm{I}_{t o}$ densities

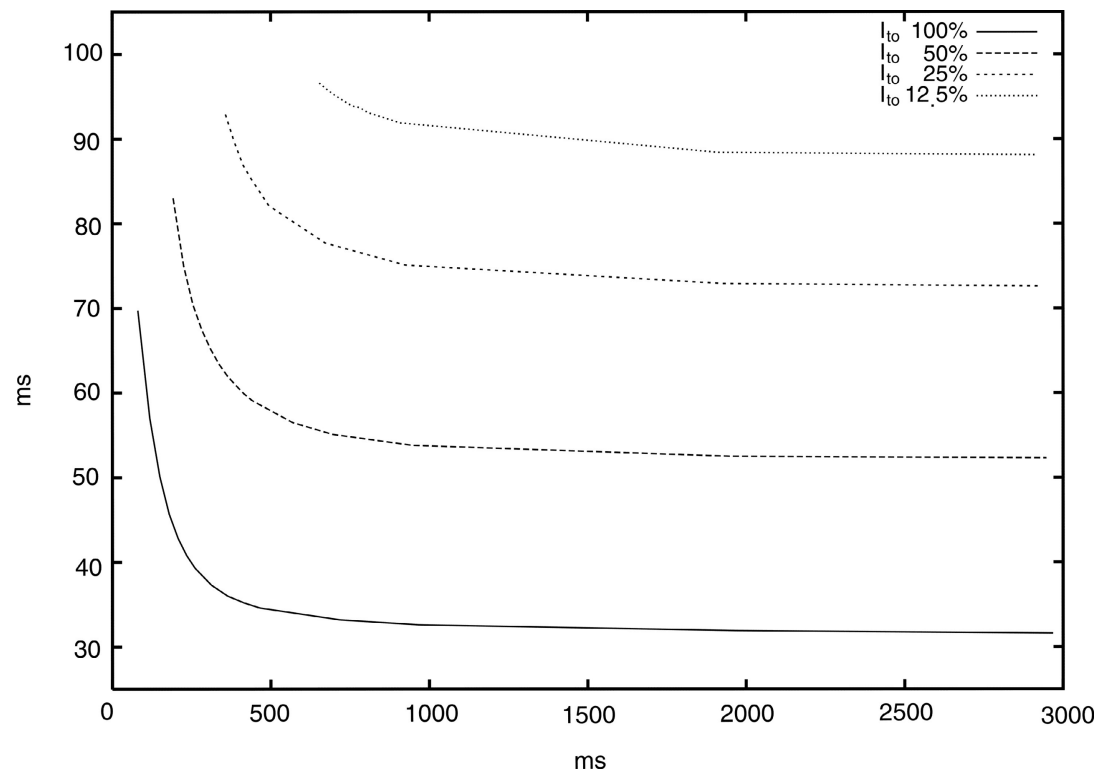

Fig. 3. Epicardial APD for different DIs and $I_{t o}$ densities 


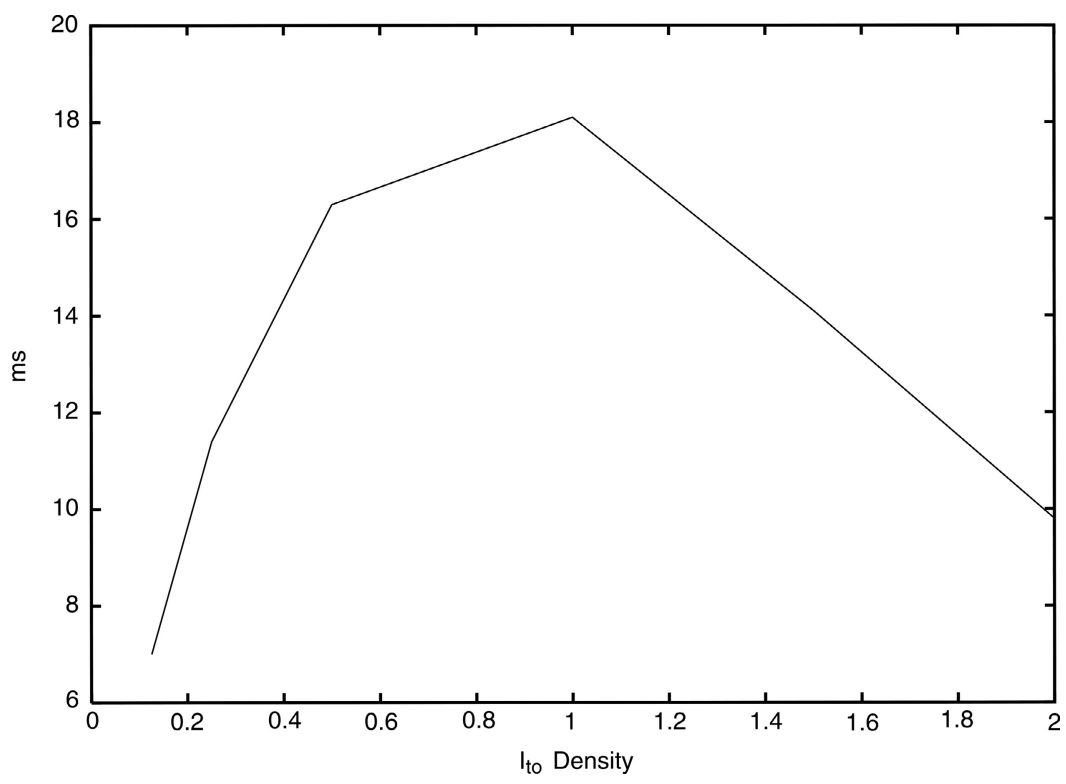

Fig. 4. Difference between endocardial and epicardial APDs for different $I_{t o}$ densities

We have also analyzed the dynamics of intracellular $\mathrm{Ca}^{2+}$ concentration by using the same steady-state restitution protocol described above. Calcium intracellular concentration was also recorded during 100 pulses at a constant BCL of $750 \mathrm{~ms}$. The density of the $\mathrm{I}_{t o}$ current was reduced to $50 \%, 25 \%$ and $12.5 \%$ in order to investigate the occurrence of EAD and its relation to APD. Transmembranic currents and ion concentrations were computed, stored, visualized and used in subsequent analysis.

\section{$3 \quad$ Results}

\subsection{Restitution Curves and EADs}

Experiments with cardiac myocytes in the acute stage of Chagas' Disease [4] indicate that phase 1 repolarization is attenuated and suggest a depression of $\mathrm{I}_{t o}$. In this work we investigated the influence of $\mathrm{I}_{t o}$ in the APD restitution. For this task we changed the maximum conductance of the $\mathrm{I}_{t o}$ current varying its density from $12.5 \%$ to $100 \%$ (control condition).

Figure 2 shows APs using the Modified Pandit Model paced at a BCL of $250 \mathrm{~ms}$ for both control $\left(100 \%\right.$ of $\left.\mathrm{I}_{t o}\right)$ and $\mathrm{I}_{t o}$ reduced to $50 \%$ conditions. At this BCL, Figure 2 shows that a pattern similar to EAD appears after $9 \mathrm{~s}$ of simulation when $\mathrm{I}_{t o}$ is reduced. At BCLs longer than $275 \mathrm{~ms}$ we have not observed EADs, i.e. with $\mathrm{I}_{t o}$ reduced to $50 \%$. However, after further reducing $\mathrm{I}_{t o}$ density to $25 \%(12.5 \%)$ EAD-like patterns show up again for BCLs shorter than $450 \mathrm{~ms}$ 
(750 ms). Therefore, these simulations suggest that the reduction of $\mathrm{I}_{t o}$ density increases the probability of EAD occurrence at physiological rates.

APD restitution curves were calculated and are presented in Figure 3, where APD is plotted versus the diastolic interval (DI). DI was taken as the difference of the BCL and the measured APD90. One may note from Figure 3 that APD decreases for longer BCLs reaching a steady-state condition after $1000 \mathrm{~ms}$. In addition, reducing the maximum conductivity of $\mathrm{I}_{t o}$ monotononically increases APD. The APD restitution curve may be used as an indication of pro-arrhythmic behavior. During pacing and reentrant cardiac arrhythmias, APD restitution slope has been shown to be an important determinant of wave stability. In addition to promoting APD alternans, a steep (modulus greater than 1) APD restitution slope can promote breakup of electrical waves into a fibrillation-like state [11. The slopes of the restitution curves presented by Figure 3 were significantly smaller than 1. This agrees with the fact that alternans were not observed in any of the simulation results.

\subsection{Dispersion of Repolarization}

It is well known that amplification of dispersion of repolarization underlies the development of life-threatening ventricular arrhythmias 12. The dispersion of repolarization is influenced by the dispersion of APDs of the ventricular myocytes and the propagation velocity of the depolarization wave. In this work we have taken the difference between endocardial and epicardial APDs as an indication of transmural APD dispersion. In addition, since the propagation velocity is proportional to the maximum rise of the action potential, we measured $\max d v / d t$ and considered this as an indication of changes on the propagation velocity.

Figure 4 presents the difference of APDs between simulated endocardial and epicardial cells for different densities of $\mathrm{I}_{t o}$ with a pacing rate of $1 \mathrm{~Hz}$. Note the biphasic behavior. APD dispersion is greatly decreased by reducing $\mathrm{I}_{t o}$ density (a near 4-fold decrease from control to $12.5 \%$ of $\mathrm{I}_{t o}$ density). However, increasing $\mathrm{I}_{t o}$ beyond the control level also reduces APD dispersion.

Since $\max d v / d t$ is related with the velocity of the wave propagation in the cardiac tissue, we simulated the influence of the $\mathrm{I}_{t o}$ in the upstroke of the AP. Figure 5 shows that the longer the cycle length the greater the upstroke. Figure 5 also shows that $\mathrm{I}_{t o}$ marginally affects $\max d v / d t$. The reduction of $\mathrm{I}_{t o}$ was followed by a $10 \%$ decrease of $\max d v / d t$.

\subsection{Changes in the Intracellular $\mathrm{Ca}^{2+}$ Concentration}

Alterations in intracellular calcium handling play a prominent role in the generation of arrhythmias in the failing heart [13. Figure 6 presents the $\left[\mathrm{Ca}^{2+}\right] \mathrm{i}$ versus time for a BCL of $750 \mathrm{~ms}$. By reducing $\mathrm{I}_{t o}$ density we observe that $\left[\mathrm{Ca}^{2+}\right] \mathrm{i}$ reaches a larger steady-state value. The results thus suggest that reduction of $\mathrm{I}_{t o}$ density is followed by $\left[\mathrm{Ca}^{2+}\right] \mathrm{i}$ overload. 


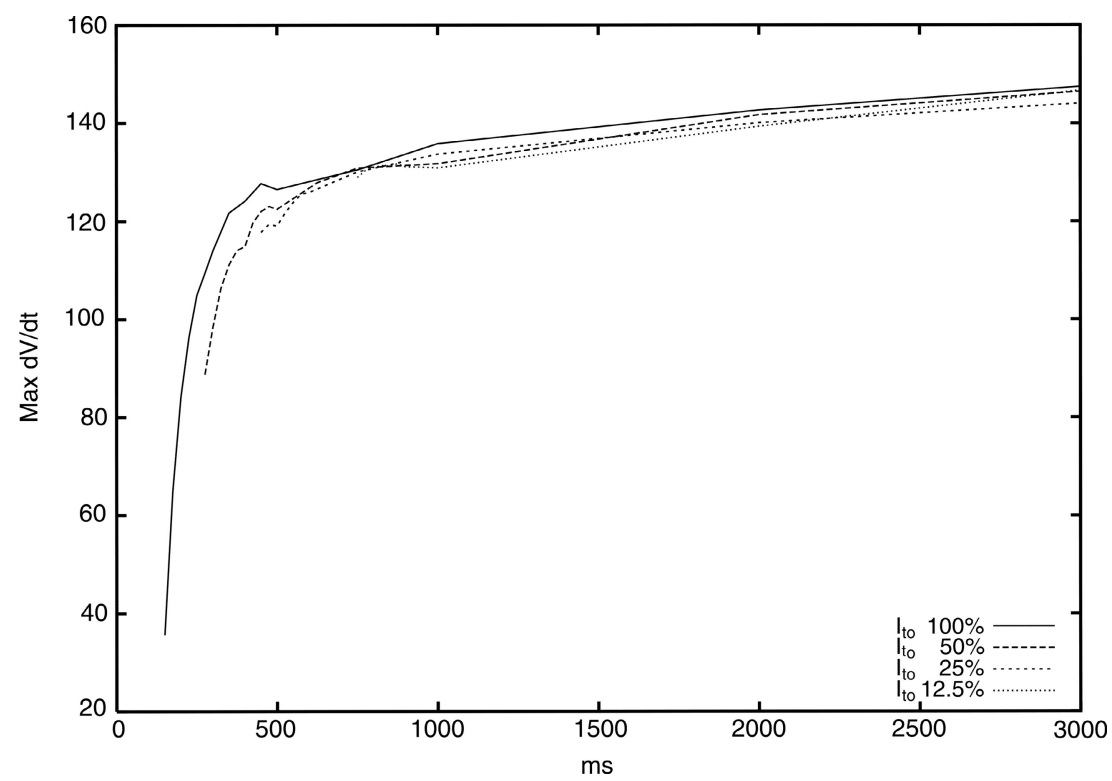

Fig. 5. The upstroke velocity $\max d v / d t$ for different DIs

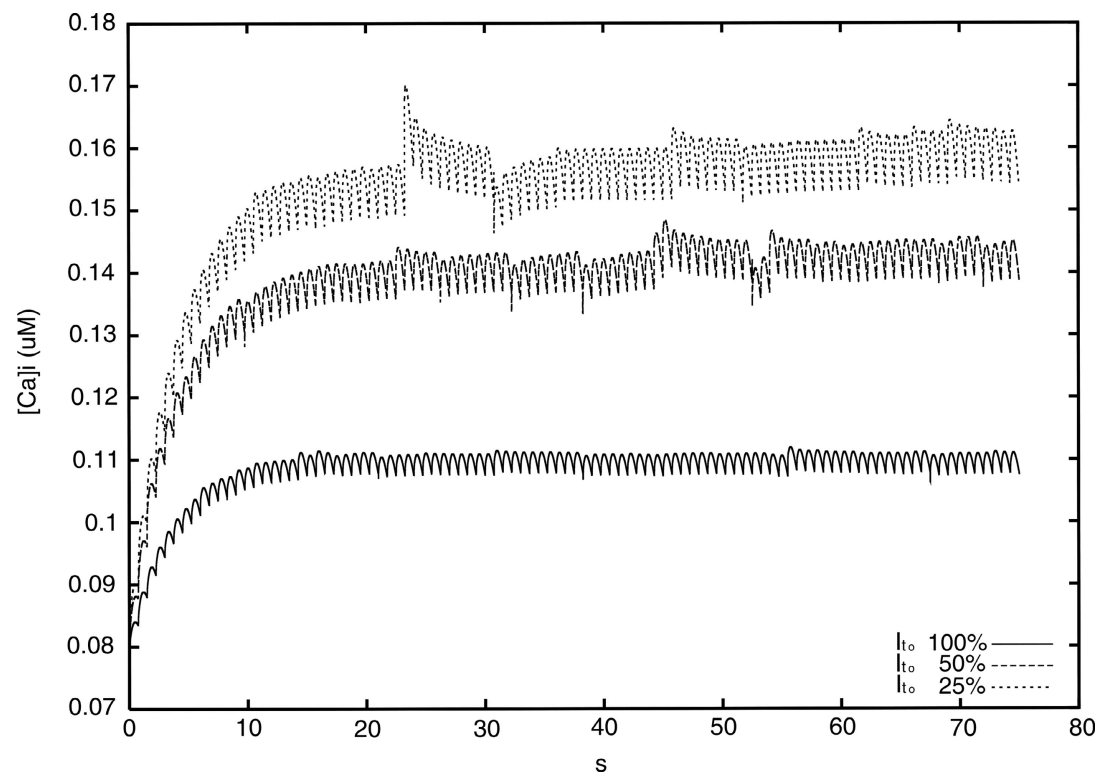

Fig. 6. $\left[\mathrm{Ca}^{2+}\right]$ i for different densities of $\mathrm{I}_{t o}$ versus time 


\section{Discussion}

In this work we have used a Web-based cardiac computational framework based on the CellML language to simulate mathematical models of the left ventricular myocytes of adult rats. The simulations seek a better understanding of the effects of $\mathrm{I}_{t o}$ reduction during the acute phase of Chagas' Disease. APD restitution curves were generated, the transmural dispersion of repolarization was investigated and the dynamics of $\left[\mathrm{Ca}^{2+}\right] \mathrm{i}$ analyzed. We have not observed the phenomenon of alternans during the steady-state pacing protocol adopted. The APD restitution curves were calculated as presented in Figure 3 It is known that a steep APD restitution slope may suggest the breakup of electrical waves into a fibrillation-like state. The absolute values of the slopes of the restitution curves presented by Figure 3 were significantly smaller than 1 . This agrees with the fact that alternans were not observed in any of the simulation results.

However, during fast pacing a pattern similar to Early Afterdepolarization (EAD) was present (see Figure 21). In addition, EADs occurred in considerably slower pacing rates after $\mathrm{I}_{t o}$ density was reduced in the models. Therefore, the results suggest that the reduction of $\mathrm{I}_{t o}$ density increases the probability of EAD occurrence at physiological rates. The premature occurrence of EADs seems to be in accordance with the accumulation of $\left[\mathrm{Ca}^{2+}\right] \mathrm{i}$ as a consequence of reduction of $\mathrm{I}_{t o}$ density (see Figure 6). Calcium entry into myocytes drives myocyte contraction. To prepare for the next contraction, myocytes must extrude calcium from intracellular space via the $\mathrm{Na}^{+} / \mathrm{Ca}^{2+}$ exchanger (NCX1) or sequester it into the sarcoplasmic reticulum. Defective calcium extrusion correlates with increased intracellular calcium levels and may be relevant to heart failure and to the generation of arrhythmias [19. The results presented in Figure 6 suggest that reduction of $\mathrm{I}_{t o}$ density may be followed by $\left[\mathrm{Ca}^{2+}\right] \mathrm{i}$ overload.

We have addressed the effects of $\mathrm{I}_{t o}$ density reduction on the transmural dispersion of repolarization by calculating the changes on endocardial to epicardial APD difference and on the $\max d v / d t$, which is related to the velocity of the wave propagation in the cardiac tissue. In our simulations, max $d v / d t$ was marginally affected (see Figure 4). However, the transmural dispersion of APD, as presented in Figure 5 , is decreased after $\mathrm{I}_{t o}$ is reduced. The results indicate an anti-arrhythmic effect associated to the reduction of $\mathrm{I}_{t o}$ density.

In summary, the computational framework simplified the required modifications and usage of the mathematical model studied in this work. The combination of the XML-based automatic code generator, the CellML editor and the Web portal provided an user-friendly environment for our simulations. Our results suggest that the reduction of $\mathrm{I}_{t o}$ density observed during the acute phase of Chagas' Disease elicits the modification of many important electrophysiological mechanisms and features. The reduction of $\mathrm{I}_{t o}$ was followed by $\left[\mathrm{Ca}^{2+}\right] \mathrm{i}$ overload and promoted the occurrence of EADs, acting thus in a pro-arrhythmic fashion. However, reduction of $\mathrm{I}_{t o}$ also decreased the dispersion of APDs, indicating an anti-arrhythmic effect. Therefore, the observed modifications do not converge to the same direction in what concerns the prevention or induction of arrhythmia. Further studies are necessary in order to better characterize and understand the electrophysiological changes that underly the acute phase of Chagas' Disease. 
Acknowledgments. The authors would like to thank the support provided by UFJF, CNPQ and FAPEMIG, project TEC-1358/05. Caroline Costa, Ricardo Campos and Fernando Campos are FAPEMIG scholarship holders.

\section{References}

1. Control of Chagas' Disease. Report of WHO Expert Committee. World Health Organization, Geneva, p. 1 (1991)

2. Laranja, F.S., Dias, E., Nobrega, G., Miranda, A.: Chagas' Disease. A clinical, epidemiologic and pathologic study. Circulation 14, 1035-1060 (1956)

3. Rosenbaum, M.B.: Chagasic myocardiopathy. Prog. Cardiovasc. Dis. 7, 199-225 (1964)

4. Pacioretty, L.M., Barr, S.C., Han, W.P., Gilmour Jr., R.F.: Reduction of the transient outward potassium current in a canine model of Chagas' Disease. Am. J. Physiol. 268(3 Pt 2), H1258-H1264 (1995)

5. Rat Genome Sequencing Project Consortium: Genome sequence of the Brown Norway rat yields insights in to mammalian evolution. Nature 428, 493-521 (2004)

6. Abbot, A.: The renaissance rat. Nature 428, 464-466 (2004)

7. Barbosa, C.B., Santos, R.W., Amorim, R.M., Ciuffo, L.N., Manfroi, F.M., Oliveira, R.S., Campos, F.O.: A Transformation Tool for ODE based models. In: Alexandrov, V.N., van Albada, G.D., Sloot, P.M.A., Dongarra, J. (eds.) ICCS 2006. LNCS, vol. 3991, pp. 69-75. Springer, Heidelberg (2006)

8. Martins, D., Campos, F.O., Ciuffo, L.N., Oliveira, R.S., Amorim, R.M., Vieira, V.F., Ebecken, N.F.F., Barbosa, C.B., Santos, R.W.: A Computational Framework for Cardiac Modeling Based on Distributed Computing and Web Applications. In: Daydé, M., Palma, J.M.L.M., Coutinho, Á.L.G.A., Pacitti, E., Lopes, J.C. (eds.) VECPAR 2006. LNCS, vol. 4395, pp. 544-555. Springer, Heidelberg (2007)

9. CellMl 1.1 (2006), http://www.cellml.org/specifications/cellml_1.1/

10. Laboratory of Computational Physiology, http://www.fisiocomp.ufjf.br

11. Zemlin, C.W., Panfilov, A.V.: Spiral waves in excitable media with negative restitution. Phys. Rev. E Stat. Nonlin. Soft Matter. Phys. 63(4 Pt 1), 041912 (2001)

12. Antzelevitch, C.: Role of spatial dispersion of repolarization in inherited and acquired sudden cardiac death syndromes. Am. J. Physiol. Heart Circ. Physiol. 293(4), H2024-H2038 (2007)

13. Burashnikov, A., Antzelevitch, C.: Late-phase 3 EAD. A unique mechanism contributing to initiation of atrial fibrillation. Pacing Clin. Electrophysiol. 29(3), 290295 (2006)

14. Pandit, S.V., Clark, R.B., Giles, W.R., Demir, S.S.: A mathematical model of action potential heterogeneity in adult rat left ventricular myocytes. Biophys. J. 81(6), 3029-3051 (2001)

15. CellMl Repository, http://www.cellml.org/models/

16. Kondratyev, A.A., Ponard, J.G., Munteanu, A., Rohr, S., Kucera, J.P.: Dynamic changes of cardiac conduction during rapid pacing. Am. J. Physiol. Heart Circ. Physiol. 292(4), H1796-H1811 (2007)

17. SGE, The Sun Grid Engine, http://gridengine.sunsource.net/

18. Koller, M.L., Riccio, M.L., Gilmour Jr., R.F.: Dynamic restitution of action potential duration during electrical alternans and ventricular fibrillation. Am. J. Physiol. 275(5 Pt 2), H1635-H1642 (1998)

19. Bers, D.M., Despa, S., Bossuyt, J.: Regulation of $\mathrm{Ca} 2+$ and $\mathrm{Na}+$ in normal and failing cardiac myocytes. Ann. N Y Acad. Sci. 1080, 165-177 (2006) 